\title{
Detection of Enteroviral RNA in Idiopathic Dilated Cardiomyopathy and Other Human Cardiac Tissues
}

\author{
Lawrence M. Weiss, Xian-Fang Liu, Karen L. Chang, and Margaret E. Billingham \\ Departments of Pathology, City of Hope National Medical Center, Duarte, California 91010-0269; \\ and Stanford University, Stanford, California 94305
}

\begin{abstract}
Enteroviruses have been considered to be a possible cause of idiopathic dilated cardiomyopathy. We used a polymerase chain reaction methodology for the identification of enteroviral RNA in an attempt to provide evidence of a role for enteroviruses in the pathogenesis of idiopathic dilated cardiomyopathy. The methodology was shown to identify a wide variety of enteroviruses with a sensitivity up to 0.1-1 plaque-forming units / gram of tissue. 5 of 11 cases (45\%) of idiopathic dilated cardiomyopathy, as well as 9 of 24 cases (38\%) of a wide variety of other cardiac conditions (including normal heart), were positive for enteroviral nucleic acid sequences; all eight control cases of breast carcinoma tested were negative. These results suggest that both the normal and abnormal heart may represent a site of latent or low-grade persistent enteroviral infection, and that the mere presence of enteroviral nucleic acid sequences is not specifically associated with idiopathic dilated cardiomyopathy. (J. Clin. Invest. 1992. 90:156-159.) Key words: polymerase chain reaction $\bullet$ coxsackievirus $\bullet$ thyrology $\bullet$ idiopathic dilated cardiomyopathy $\bullet$ enterovirus
\end{abstract}

\section{Introduction}

Idiopathic dilated cardiomyopathy (IDC) ${ }^{1}$ is a pathologic process characterized grossly by dilatation of the ventricles and histologically by marked myocyte hypertrophy with myocytes containing large bizarre nuclei, extensive interstitial fibrosis, focal replacement fibrosis, and endocardial thickening $(1,2)$. It is an end-stage process and, by definition, has no known etiology. However, many investigators favor the possibility that IDC most often results as a sequela to acute viral myocarditis, either caused by persistence of virus or as a result of an autoimmune phenomenon secondary to previous exposure to virus. Evidence in favor of this hypothesis includes the presence of foci of inflammatory cells in IDC, the difficulty in both the clinical and pathologic distinction between IDC and acute

Address correspondence and reprint requests to Lawrence M. Weiss, M.D., Department of Pathology, City of Hope Medical Center, 1500 East Duarte Road, Duarte, CA 91010-0269.

Received for publication 6 September 1991 and in revised form 5 February 1992.

1. Abbreviations used in this paper: IDC, idiopathic dilated cardiomyopathy; PCR, polymerase chain reaction.

J. Clin. Invest.

(c) The American Society for Clinical Investigation, Inc.

0021-9738/92/07/0156/04 \$2.00

Volume 90, July 1992, 156-159 myocarditis, and data obtained from murine models of myocarditis (1, 3-7).

Since enteroviruses are thought to be the most common cause of acute myocarditis, several groups have sought evidence of these viruses in IDC. Two groups have obtained evidence of enterovirus in IDC using slot-blot and in situ hybridization (8-10). Recently, we and others have examined heart samples from patients with IDC using PCR (polymerase chain reaction $)(11,12)$. Jin et al. identified enteroviral RNA in 5 of 48 patients with clinically suspected myocarditis or IDC, including 3 of 20 patients with congestive or dilated cardiomyopathy (11). In our previous study, we were unable to identify viral sequences in 11 cases of IDC examined (12). One possible explanation for our negative findings is the fact that we used a combination of primers and probe specific for coxsackievirus B3, chosen because B3 has been most closely linked to acute myocarditis (13).

Recently, Chapman et al. reported use of a set of PCR primers and probes from the highly conserved $5^{\prime}$ nontranslated region of enteroviral RNA that have been shown to identify a wide range of enteroviruses (14). In the current report, we have used these oligonucleotides to identify enteroviral RNA sequences from a wide range of myocardial specimens.

\section{Methods}

Case selection. Samples were studied from one animal model, viral-infected cell lysates, and tissues obtained from human hearts. In the animal model (specimens donated by Charles J. Gauntt, PhD, San Antonio, TX), adolescent CD-1 mice were killed $3 \mathrm{~d}$ after inoculation with the virus (15). The hearts were removed, washed in Dulbecco's PBS, flash frozen in liquid nitrogen, and stored at $-20^{\circ} \mathrm{C}$ until use. The strain of coxsackievirus B3 inoculated, CVB3m, was derived from the experimental passage of the Nancy strain by E. D. Kilbourne and J. F. Woodruff at Cornell University Medical College, New York, NY. For production of infected cell lysates, cultured HeLa cells were challenged with 100-200 plaque-forming units of echoviruses $2,4,6$, or 22 , or coxsackieviruses A21 or B4.

The human specimens studied consisted of portions of tissue taken at the time of heart or heart-lung allograft transplantation. The investigation was performed after approval by the Human Subjects Committee at Stanford University. The specimens were obtained fresh and frozen at $-20^{\circ} \mathrm{C}$ until use. There were 11 cases of IDC. The duration of symptoms were $1 \mathrm{mo}, 1 \mathrm{mo}, 2 \mathrm{mo}, 2 \mathrm{mo}, 7 \mathrm{mo}, 8 \mathrm{mo}, 12 \mathrm{mo}, 36 \mathrm{mo}, 56$ $\mathrm{mo}$, and $168 \mathrm{mo}$ in the 10 cases in which this information was available. All of the cases had the classic histologic features of IDC outlined in the introduction; in none of these cases was there significant inflammation to suggest a diagnosis of myocarditis. In addition, there were nine cases of coronary artery disease, six cases of explanted heart taken at the time of heart-lung transplantation for lung disease, four cases of allograft rejection, one case obtained from a presumably normal donor heart, one case of valvular disease, one case of congenital heart disease, one case of cardiac hypertrophy, and one case of fibroelastosis. All of 
the human heart specimens had been found to be negative for coxsackievirus B3, using a highly sensitive PCR analysis specific for that serotype, as previously reported (12). In addition, eight specimens from patients with breast carcinomas and nine specimens from patients with gastric carcinomas were studied as negative controls.

Oligonucleotides. All oligonucleotides were purchased from Operon Technologies Inc., Alameda, CA. The oligonucleotides used for the panenterovirus $P C R$ studies were the $E 1$ and $E 2$ primers and E3 probe reported by Chapman et al. (14). They are derived from sequences from the highly conserved $5^{\prime}$ nontranslated region of the enterovirus genome. The oligonucleotides used for the control actin studies were those previously reported by Ben-Ezra et al. (16).

$P C R$ procedures. RNA was extracted from specimens by standard methods (17). Two micrograms of purified cellular RNA were converted to single-stranded cDNA using the E1 and E2 oligonucleotide, Moloney-MLV reverse transcriptase (Bethesda Research Laboratories, Gaithersburg, MD) and reaction conditions as described previously (18). The methods for the panenterovirus PCR studies were those previously reported by Chapman et al. (14). At least four "blank" (water substituted for template) negative controls were included in each run.

After PCR was performed, $20 \mu \mathrm{l}$ of the reaction product was run on an agarose gel. The results of the actin PCR studies were evaluated by ultraviolet light illumination of the ethidium bromide-stained gel. The reaction products from the panenterovirus PCR were analyzed by Southern blot hybridization using the E3 oligonucleotide probe end radiolabeled with $\alpha\left[{ }^{32} \mathrm{P}\right]$ adenosine triphosphate using hybridization and wash conditions described previously (19).

\section{Results}

We first sought to verify the sensitivity and specificity of the panenteroviral PCR primers and probe. As illustrated in Fig. 1, PCR performed using the panenteroviral primers and probe detected a strong signal at the expected 196 bp in RNA extracted from tissue obtained from the coxsackievirus B3-infected CD-1 mouse model. Serial dilution studies revealed an observable signal down to $10^{6}$ dilution. Because there were about $1 \times 10^{5}$ to $1 \times 10^{7}$ plaque-forming units of virus per gram of tissue in CD-1 murine hearts $3 \mathrm{~d}$ after inoculation (12), these studies establish the sensitivity of the technique at $\sim 0.1-10$ plaque-forming units of virus per gram of tissue.

To verify the specificity of the technique, PCR analyses were performed on RNA extracted from infected cell lysates of HeLa cells challenged with a variety of enteroviruses, including echoviruses 2, 4, 6, and 22, and coxsackieviruses A21, B4. As seen in Fig. 2, all of the virally infected specimens were positive using the panenteroviral primers, with the exception of echovirus 22. These findings are similar to the results of Chapman et al., who were able to amplify cDNA from 41 out of 42 differ-

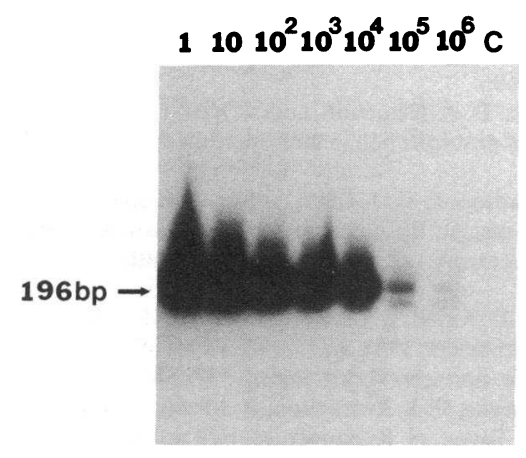

Figure 1. Results of Southern blot hybridization of the products of enteroviral PCR in serial dilution studies of coxsackievirus B3-infected heart tissue. $C$ is a water negative control.

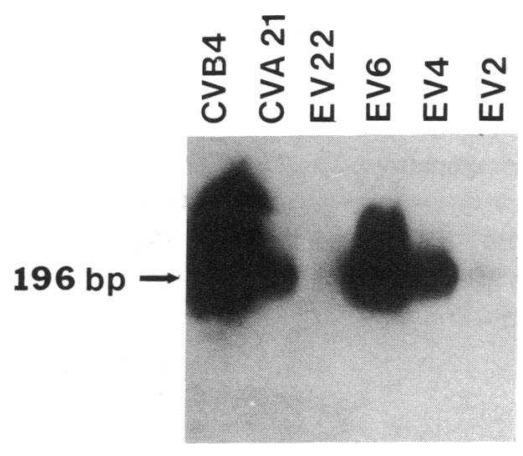

Figure 2. Results of Southern blot hybridization of the products of enteroviral PCR on six different cell lysates infected with coxsackievirus $(\mathrm{CV}) \mathrm{B} 4$ and $\mathrm{A} 21$ and echovirus (EV) 2 , 4,6 , and 22 .

ent enterovirus serotypes, with the lone exception being echovirus 22, a serotype thought to be atypical of the enteroviral genomic paradigm $(14,20)$.

11 cases of IDC and 24 myocardial specimens with a wide variety of other diagnoses were studied by PCR using the panenteroviral primers and probe; all of the specimens were shown to have adequate amounts of amplifiable RNA by PCR analyses with actin primers (Fig. 3). Overall, 14 of the 35 specimens (20\%) were positive for enteroviral nucleic acid sequences ( Táble I and Fig. 3). 5 of 11 (44\%) of the IDC cases and 9 of 24 $(38 \%)$ of the other cases were positive. There was no correlation between diagnosis and positivity, as cases of coronary artery disease, rejection, fibroelastosis, explanted hearts at the time of heart-lung transplantation for lung disease, and even a donor heart were positive. In addition, there was no correlation between diagnosis and intensity of the hybridization signal. The eight cases of breast carcinoma and nine cases of gastric carcinoma were all negative for enteroviral nucleic acid se-

\section{$\begin{array}{llllllllll}1 & 2 & 3 & 4 & 5 & 6 & 7 & 8 & 9 & 10\end{array}$}

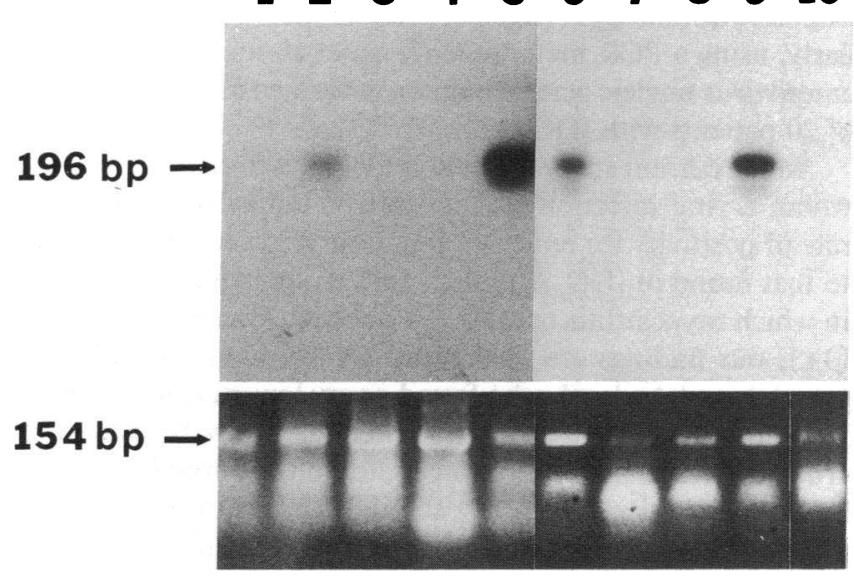

Figure 3. Results of Southern blot hybridization of the products of enteroviral PCR on 10 cardiac specimens. A composite autoradiogram from two separate experiments is shown. Lanes 1-5 represent cases of IDC. Lanes 6, 7, and 10 are cases of coronary artery disease, lane 8 is a case of cardiac hypertrophy and lane 9 is a case of an explanted heart taken at the time of heart-lung transplantation for lung disease. Shown at the bottom of the figure is a picture of an ethidium bromide-stained gel after electrophoresis of the products of PCR for actin RNA performed on the same cases; all samples show a band at $154 \mathrm{bp}$, indicating sufficient extracted RNA. 
Table I. Specimens Positive for Enteroviral Nucleic Acid Sequences

\begin{tabular}{lc}
\hline Diagnosis & \\
\hline Idiopathic dilated cardiomyopathy & $5 / 11(45 \%)$ \\
Other diagnoses & $9 / 24(38 \%)$ \\
Coronary artery disease & $3 / 9(33 \%)$ \\
Recipient heart taken at time of & \\
$\quad$ heart-lung transplantation for & \\
$\quad$ lung disease & $3 / 6 \quad(50 \%)$ \\
Allograft rejection & $1 / 4(25 \%)$ \\
Normal donor heart & $1 / 1 \quad(100 \%)$ \\
Valvular disease & $0 / 1 \quad(0 \%)$ \\
Congenital heart disease & $0 / 1 \quad(0 \%)$ \\
Cardiac hypertrophy & $0 / 1 \quad(0 \%)$ \\
Fibroelastosis & $1 / 1 \quad(100 \%)$ \\
Total & $14 / 35(40 \%)$ \\
\end{tabular}

quences (data not shown). In addition, the "blank" negative control samples were always negative.

\section{Discussion}

Using PCR methodology, we have identified enterovirus nucleic acid sequences in $45 \%$ of IDC cases. These findings are similar to the findings in the slot-blot studies and in situ hybridization studies of Bowles et al., who found virus-specific RNA sequences in tissue from approximately one-third to $40 \%$ of patients with IDC (8-10). Although it was first believed that the methodology of Bowles was specific for coxsackievirus B sequences, it is now generally acknowledged that the studies using a genomic CDNA probe derived from the coxsackievirus B2 genome detect a wide range of enteroviruses because of the extensive homology between the viruses within the family. Similarly, using a PCR methodology, Jin et al. found evidence of enterovirus nucleic acid sequences in cardiac tissue from 3 out of 20 patients with IDC (11).

In the current study, we analyzed tissues from 24 patients in whom a viral infection was considered unlikely, and found a rate of positivity for enteroviral nucleic acid sequences similar to that found in IDC. Although Jin's study included few cases in which myocarditis or IDC had not been clinically suspected (11), our findings are in contrast to the previous studies by Kandolf and Archard, who found a very low incidence of positivity in controls $(8-10)$. The differences may be caused by true differences in the incidence rates in the populations studied, or alternatively, may be caused by the exquisite sensitivity of PCR as compared to the other techniques. Since some of the positive results were obtained in patients with known cardiac muscle abnormalities, it is possible that these abnormalities may predispose to enterovirus infection. However, positive results were also obtained in patients who had presumably normal hearts (e.g., a donor heart). Therefore, it is unlikely that the mere presence of enterovirus can be equated with disease. It is possible that an enterovirus causes an initial infection, either symptomatic or asymptomatic, and then persists in the hearts of some patients, either in a latent form or as a subclinical persistent infection. Although latent infection is not a well-re- cognized characteristic of enteroviruses, the natural history of enterovirus infection in man is still not completely understood; PCR technology, with its ability to detect extremely low levels of virus, should aid in elucidating the pathophysiology of these viruses.

We cannot rule out the possibility that the enterovirus (or enteroviruses) identified in the cases of IDC is distinct and differs in pathogenicity from another enterovirus (or enteroviruses) accounting for the positive signals in the non-IDC cases. This hypothesis might explain differences in disease manifestations among the various positive cases. However, the 11 cases of IDC reported here were previously studied by a sensitive and specific PCR methodology for evidence of coxsackievirus B3, the virus suspected to be the most frequent cause of acute viral myocarditis, and no evidence of this virus was found in any of the cases (12). In addition, if this hypothesis is true, one still might expect the enterovirus positivity rate to be higher in the IDC group than in the non-IDC cases. Tracy et al. have outlined a strategy combining a universal enterovirus primer set with specific probes and high-criterion hybridization analysis after amplification to examine the genomic identity of closely related enteroviruses (12). This approach could specifically identify the immunotypes of the viruses in the positive cases, allowing one to determine whether a distinct type of enterovirus is present in the IDC group, as opposed to the other enterovirus positive cases.

It must also be kept in mind that the cases of IDC studied here represent end-stage IDC. It would also be of interest to study acute myocarditis or early stage IDC to determine whether a higher prevalence of positivity might be found within these populations. This evidence may still raise the possibility of a specific relationship between enterovirus and IDC.

\section{Acknowledgments}

This work was supported in part by grant HL-13108 from the National Institutes of Health.

\section{References}

1. Aretz, H. T., M. E. Billingham, W. D. Edwards, S. M. Factor, J. T. Fallon, J. J. Fenoglio, E. G. J. Olsen, and F. J. Schoen. 1986. Myocarditis. A histopathologic definition and classification. Am. J. Card. Pathol. 1:3-14.

2. Roberts, W. C., and V. J. Ferrans. 1975. Pathologic anatomy of the cardiomyopathies. Hum. Pathol. 6:287-342.

3. Tazelaar, H., and M. E. Billingham. 1986. Leukocytic infiltrates in idiopathic dilated cardiomyopathy: a source of confusion with active myocarditis. Am. J. Surg. Pathol. 10:405-412.

4. Kawai, C., A. Matsumori, Y. Kitawa, and T. Takatsu. 1978. Virus and the heart: viral myocarditis and cardiomyopathy. Prog. Cardiol. 7:141-162.

5. Abelman, W. H. 1973. Viral myocarditis and its sequelae. Annu. Rev. Med. 24:145-152.

6. Cambridge, G., C. G. C. Mac Arthur, A. P. Waterson, J. F. Goodwin, and C. M. Oakley. 1979. Antibodies to coxsackie B viruses in congestive heart cardiomyopathy. Br. Heart J. 41:692-696.

7. Rose, N. R., A. Herskowitz, D. A. Neumann, and N. Neu. 1988. Autoimmune myocarditis: a paradigm of post-infection autoimmune disease. Immunol. Today. 9:117-119.

8. Bowles, N. E., P. J. Richardson, E. G. J. Olsen, and L. C. Archard. 1987. Detection of coxsackie-B-virus-specific RNA sequences in myocardial biopsy samples from patients with myocarditis and dilated cardiomyopathy. Lancet. i: $1120-1123$.

9. Kandolf, R., P. Kirschner, D. Ameis, A. Canu, E. Erdmann, H. P. Schultheiss, B. Kemkes, and P. H. Hofschneider. 1988. In New Concepts in Viral Heart Disease. H.-P. Schultheiss, editor. Springer-Verlag, Berlin. 337-348.

10. Archard, L. C., C. A. Freeke, P. J. Richardson, B. Meany, E. Olsen, P. Morgan-Capner, M. L. Rose, P. Taylor, N. R. Banner, M. H. Yacoub, and N. E. 
Bowles. 1988. In New Concepts in Viral Heart Disease. H.-P. Schultheiss, editor. Springer-Verlag, Berlin. 359-362.

11. Jin, O., M. J. Sole, J. W. Butany, W.-K. Chia, P. R. McLaughlin, P. Liu, and C. C. Liew. 1990. Detection of enterovirus RNA in myocardial biopsies from patients with myocarditis and cardiomyopathy using gene amplification by polymerase chain reaction. Circulation. 82:8-16.

12. Weiss, L. M., L. A. Movahed, and M. E. Billingham. 1991. Detection of coxsackievirus B3 RNA in myocardial tissues by the polymerase chain reaction. Am. J. Pathol. 138:497-503.

13. Woodruff, J. F. 1980. Viral myocarditis. A review. Am. J. Pathol. 101:427-529.

14. Chapman, N. M., S. Tracy, C. J. Gauntt, and U. Fortmueller. 1990. Molecular detection and identification of enteroviruses using enzymatic amplification and nucleic acid hybridization. J. Clin. Microbiol. 28:843-850.

15. Gauntt, C. J., P. T. Gomez, P. S. Duffey, J. A. Grant, W. E. Trent, S. M Witherspoon, and R. E. Paque. 1984. Characterization and myocarditic capabilities of coxsackievirus B3 variants in selected mouse strains. J. Virol. 52:598-605.
16. Ben-Ezra, J., D. A. Johnson, J. Rossi, N. Cook, and A. Wu. 1991. Effect of fixation on the amplification of nucleic acids from paraffin-embedded material by the polymerase chain reaction. J. Histochem. Cytochem. 39:351-354.

17. Chomcszynski, P., and N. Sacchi. 1987. Single-step method of RNA isolation by acid guanidinium thiocyanate-phenol-chloroform extraction. Anal. Biochem. 162:156-159.

18. Kohler, S., N. Galili, J. Sklar, K. G. Blume, and M. L. Cleary. 1990. Expression of $b c r-a b l$ fusion transcripts following bone marrow transplantation for Philadelphia chromosome-positive leukemia. Leukemia (Baltimore). 4:541547.

19. Ngan, B., J. Nourse, and M. L. Cleary. 1989. Detection of chromosomal translocation $t(14 ; 18)$ within the minor cluster region of $b c l-2$ by polymerase chain reaction and direct genomic sequencing of the enzymatically amplified DNA in follicular lymphomas. Blood. 73:1759-1762.

20. Rotbart, H. A., P. S. Eastman, J. L. Ruth, K. K. Hirata, and M. J. Levin. 1988. Nonisotopic oligomeric probes for the human enteroviruses. J. Clin. Microbiol. 26:2669-2671. 